\title{
BMJ Open Breast cancer application protocol: a randomised controlled trial to evaluate a self-management app for breast cancer survivors
}

\author{
Andy S K Cheng (D , , Xiangyu Liu, ${ }^{2}$ Peter H F Ng, ${ }^{3}$ Cindy T T Kwok, ${ }^{1}$ \\ Yingchun Zeng (D) , ${ }^{4}$ Michael Feuerstein ${ }^{5,6}$
}

To cite: Cheng ASK, Liu X, $\mathrm{Ng}$ PHF, et al. Breast cancer application protocol: a randomised controlled trial to evaluate a selfmanagement app for breast cancer survivors. BMJ Open 2020;10:e034655. doi:10.1136/ bmjopen-2019-034655

- Prepublication history for this paper is available online. To view these files, please visit the journal online (http://dx.doi. org/10.1136/bmjopen-2019034655).

Received 05 0ctober 2019 Revised 09 May 2020 Accepted 12 May 2020
Check for updates

(C) Author(s) (or their employer(s)) 2020. Re-use permitted under CC BY-NC. No commercial re-use. See rights and permissions. Published by BMJ.

For numbered affiliations see end of article.

Correspondence to Dr Andy S K Cheng; andy.cheng@polyu.edu.hk

\section{ABSTRACT}

Introduction The eHealth technologies that are being designed for chronic disease constitute a global trend towards health assessment and self-management. However, most of these approaches tend to focus on a single symptom or problem rather than on the multiple problems that are characteristic of many of these chronic illnesses. The aim of this study is to examine the effectiveness of and adherence to a self-management application (app) that identifies multiple problem areas related to surviving breast cancer as the targeted chronic illness.

Methods and analysis This is a randomised controlled study. Eligible participants will be allocated randomly into either an intervention group or a control group at a $1: 1$ ratio. The intervention group will be assigned to the self-management app ('Be-with-You'), while the control group will use a general health app ('Sham' app). The primary outcomes will include the differences between the two groups in their health literacy, problem-solving skills and self-management skills. The secondary outcomes will include group differences in self-efficacy, readiness for change and health-related quality of life. All of these outcomes will be measured at baseline and at 4 weeks and 12 weeks after intervention. In addition, usability of these two mobile apps will be measured at 4 weeks and 12 weeks after intervention. The planned sample size is 476.

Ethics and dissemination The Human Subjects Ethics Sub-committee of The Hong Kong Polytechnic University approved the study (HSEARS20190922001, 24 September 2019). Dissemination of findings will occur at the local, national and international levels.

Trial registration number ChiCTR1900026244.

\section{BACKGROUND}

According to the WHO, cancer is one of the four major types of non-communicable chronic disease (NCD) and the second leading cause of NCD-related deaths worldwide. In China, breast cancer is the most frequently diagnosed female cancer, with 367900 newly diagnosed cases and 97972 deaths $^{1}$ in 2018 . However, the 5-year relative
Strengths and limitations of this study

- This study will examine the effectiveness of a selfmanagement app that identifies multiple problem areas related to breast cancer survivors.

- This study will provide independent assessment (ie, not dependent on participant reporting) of access to and use of the two apps, through the use of statistics monitored by the two apps.

- This study is not intended to determine the specific effect of each app on change in the specific problem over time. The outcome will measure the actual engagement with the two apps and the effects on self-management and general health-related quality of life.

- This study will only provide one type of specific self-management skill (use of a tool that indicates potential problem areas experienced following treatment for breast cancer and provides information for follow-up to access resources for identified problem areas).

survival rate of female breast cancer increased from $73.1 \%$ in $2003-2005$ to $82 \%$ in 2012$2015 .^{2}$ With further medical advancements, it is expected that the survival rate for women diagnosed with breast cancer will continue to increase over the next few decades. In China, the State Council has introduced a medium to long-term plan called 'Healthy China 2030' that aims at strengthening the prevention and treatment of chronic diseases, reducing the financial burden of medical expenses, improving the average life expectancy and enhancing the overall health of Chinese people by $2030 .^{3}$

Breast cancer survivors often experience a wide range of unmet needs, including symptom burden. ${ }^{4-6}$ Symptom burden is defined as the subjective, quantifiable prevalence, frequency and severity of symptoms placing a physiological burden on patients and producing multiple negative, 
physical and emotional patient responses. ${ }^{7}$ Much of the symptom burden in breast cancer survivors is related to medical treatment, such as chemotherapy, radiotherapy and mastectomy. Cognitive dysfunction, lymphoedema, fatigue, sexual dysfunction, anxiety and depressive symptoms are problems of breast cancer survivors during and following primary treatment. ${ }^{8}$ A systematic review of the literature indicates that symptoms that are present immediately after the completion of primary treatment for cancer can persist for more than 5 years. ${ }^{9}$ Those symptoms can significantly lower the survivors' quality of life and functional outcomes, including work productivity. ${ }^{10-14} \mathrm{~A}$ qualitative study has suggested that the post-treatment selfmanagement needs of breast cancer survivors comprise five major areas: symptom management needs, emotional management needs, information acquisition needs, the need for a relationship with healthcare providers and adaptation needs. ${ }^{15}$ Because symptoms and many other problems experienced by breast cancer survivors are personal and multifactorial, and because provider-based healthcare following primary treatment can often represent a challenge, patient-based self-management in terms of improving the ability to identify and seek resources provides significant assistance for enhancing the physical and psychological health and reducing potential unhealthy lifestyle often related to surviving breast cancer, and helps them to access needed comprehensive chronic healthcare that can better improve their overall quality of health.

The chronic care model (CCM) and the chronic disease self-management model Programme (CDSMP) strongly urge the use of self-management in chronic disease. The CCM comprises six interacting elements (the health system, clinical information system, decision support, delivery system design, self-management support and community resources for patients) that work together to fulfil the needs of patients with chronic illnesses. ${ }^{16}$ The nature of the CCM requires greater involvement of healthcare professionals and patients in primary care than the CDSMP does. Although existing resources, division of labour and decision-making at the level of senior management affect the implementation of the CCM, ${ }^{17}$ the model also emphasises the therapeutic alliance and collaborative decision-making among healthcare professionals, programme leaders and patients. Although the CCM has generated positive outcomes, its practicality in primary care has been challenged because front-line healthcare professionals report that high job demands leave them with minimal time for proactive care and necessary follow-up. ${ }^{17}$

The CDSMP, on the other hand, is based on the social cognitive theory, which aims at enhancing the selfefficacy of patients so that they can better manage their own disease. ${ }^{18}$ In one study, six self-management skills (problem solving, decision-making, resource utilisation, the formation of a patient-provider partnership, action planning and self-tailoring) were taught through a 6 -week group-based workshop by two trained leaders who were non-healthcare professionals and had a chronic disease. ${ }^{19}$ The CDSMP has been criticised for its use of a restricted time frame, such as the 6-week duration of the workshop. Also, trained leaders may have presuppositions that affect the delivery of the self-management information and therefore could have influenced group dynamics. ${ }^{20}$ The effectiveness of the CCM and the CDSMP on cancer survivors still remains to be definitively determined, because only a few existing studies have adopted them as interventions. $^{21}$ Patient activation and a self-management approach is a common element of a CCM approach and may assist with the time demands characteristics of primary care. The specific effect of a version of self-management directed at identifying and providing resources to address the identified problem areas as reported by patients remains unclear. ${ }^{22}$

Despite the lack of research on disease selfmanagement, eHealth technologies constitute a global trend to promote chronic disease self-management. ${ }^{23}$ The approach is suggested to be used to minimise healthcare costs while also attending to multiple reported problems, potentially reducing the need for extensive involvement of healthcare professionals. ${ }^{24}$ Recently, several mobile phone or tablet applications (apps) and web pages that target different symptom management goals, such as psychological adjustment, lymphoedema symptom management, arm and shoulder exercises, dietary management and weight management, have been specially designed for breast cancer survivors. ${ }^{25-28}$ However, most of those apps and web pages have tended to focus on the assessment and/or management of a single symptom or problem area. Because many breast cancer survivors report multiple problems, the use of many single-problem apps especially immediately following treatment can be stressful and cumbersome when identifying and managing the patients' multiple problems. BENECA mHealth app developed by LozanoLozano and colleagues has highlighted the importance of the use of mobile apps to address breast cancer survivors' multiple problems in order to motivate them to adhere to healthier lifestyles. ${ }^{29}{ }^{30}$ Moreover, systematic reviews and meta-analyses of eHealth approaches for use with cancer survivors report the need for future research that will consider the use of different approaches to multiple problem areas. ${ }^{31} 32$

The aim of this study is to examine the effectiveness of and adherence to a self-management app designed to address multiple problem areas for breast cancer survivors in a large sample. The objectives are: (1) to examine whether there is a difference in the health literacy, problem-solving skills, readiness for change, self-efficacy, self-management skills and health-related quality of life (HRQoL) between the intervention group and the control group, and (2) to examine the usability of this self-management app versus that of a general health app. We predict that there will be greater adherence to the active self-management app. We also anticipate that those using it will have greater levels of health literacy, problem-solving skills and self-efficacy, 
thus resulting in higher readiness for change, an aspect of self-management skills and overall HRQoL.

\section{METHODS}

\section{Design}

This study is to be a randomised controlled trial in which all participants are blinded to the study's two groups of app users (the intervention group and the control group) and are assigned to the groups at a 1:1 ratio by computergenerated random numbers, implemented by a research assistant. The study will be conducted in Hunan province, China, from 1 January 2020 to 30 June 2021. The participants who are allocated to the intervention group will use the self-management 'Be-with-You' app, and the participants in the control group will use a general health app.

\section{Be-with-You app}

The 'Be-with-You' app consists of two major parts. The first part is the Chinese version of the Cancer Survivor Profile for breast cancer survivors (CSPro-BC) $)^{33}$ and includes a 71-item survey that was adapted from its original psychometrically sound Cancer Survivor Profile model. ${ }^{34}{ }^{35}$ It provides a self-assessment tool that can effectively identify 18 subscales within five problem domains and will automatically generate a profile for the patient to use to visualise her current condition. Oncology nurses will also provide guidance on the use of the app, along with problem-solving training. ${ }^{35}$

The participants' profiles will be generated according to three steps. (1) Raw scores: The app will first calculate raw scores for each of the 18 independent subscales of the five problem domains: the symptom burden domain (fatigue, fear of recurrence, anxiety, pain, body image and depressive symptoms), the function domain (cognitive, social, work, sexual and sleep), the health behaviours domain (unhealthy diet and physical activity), the healthcareseeking skills domain (patient-provider communication, health information, healthcare competence and information acquisition) and the domain of financial strain related to breast cancer. (2) Standardised scores: A z-score will first be calculated based on the following formula, in which the mean and SD for each of the 18 subscales are generated, with reference to a group of 444 breast cancer survivors with a similar history of diagnosis and treatment who we had recruited in previous study. ${ }^{33}$

$$
z \text { score }=\frac{\text { raw score }- \text { mean score of previous BCS cases }}{\text { standard deviation of previous BCS cases }}
$$

Finally, a standardised score ( $t$-score) will be calculated using the formula:

$$
\text { score }=(z-\text { score } * 10)+50
$$

(3) Generalisation of the profile: Each scale in the profile will be visualised either: (1) as a potential problem area to follow-up on, (2) a value of score that is in the 'average range', or (3) a value of the score on a given scale that is 'below average' and does not need to be followed up on. Each of the three ranges is indicated by colour-based classifications: red-do something about; yellow-keep an eye on it; and green-just go about your life. ${ }^{34} 35$

The second major part of the 'Be-with-You' app is composed of self-learning e-resources, such as expert articles, video to demonstrate how to use the app, different types of exercise to improve physical functioning, stress management, weight and diet, and so on, and referrals to local community organisations and providers. The resources have been developed and content validated through focus group discussions with breast cancer survivors (BCS) themselves and other stakeholders including various types of BCS providers to obtain suggestions of people or programmes to be accessed by location and problem area, per the profile results. An app-based push notification feature will be incorporated to gently remind the participants of the need to regularly review their BCS profile and latest self-management resources (figure 1).

\section{General health app (Sham app)}

The general health app has been developed by putting together current health information that is typically given to breast cancer survivors to manage their health conditions. An app-based push notification feature will also be found in the general health app that reminds the patients to review different information that is of interest to them. Oncology nurses will also provide time to help orient the participants to this app.

Both apps will support both major mobile platforms, such as iOS and Android, and will be installed on the participants' smartphones during the first outpatient visit. In addition, both apps will automatically record usage log data, including when and what the participants log into, as well as how long they review the different contents of the app or have any other interaction with the app. By interacting with the app in these two ways, it is believed that the participants will individualise their cycle of experiential learning by performing active engagement related to their self-management. All data received from the 'Be-with-You' app and the general health app will be stored on a centralised database server that is hosted by The Hong Kong Polytechnic University and will significantly protect data privacy and will transfer data securely throughout the entire period of this proposed study.

\section{Patient and public involvement}

The e-resources of both apps have been identified using focus group discussions with BCS and various types of BCS providers coupled with the use of evidence-based resources.

\section{Eligibility criteria}

All participants will be recruited voluntarily from Hunan Cancer Hospital-The Affiliated Cancer Hospital of Xiangya School of Medicine, Central South University (http://en.hnszlyy.com/index.html). It is a provincial institute for cancer treatment and research. In 2017, the Hospital has 1490 beds. It had 16870 operations on inpatients and 372781 outpatient attendances. Each year, 

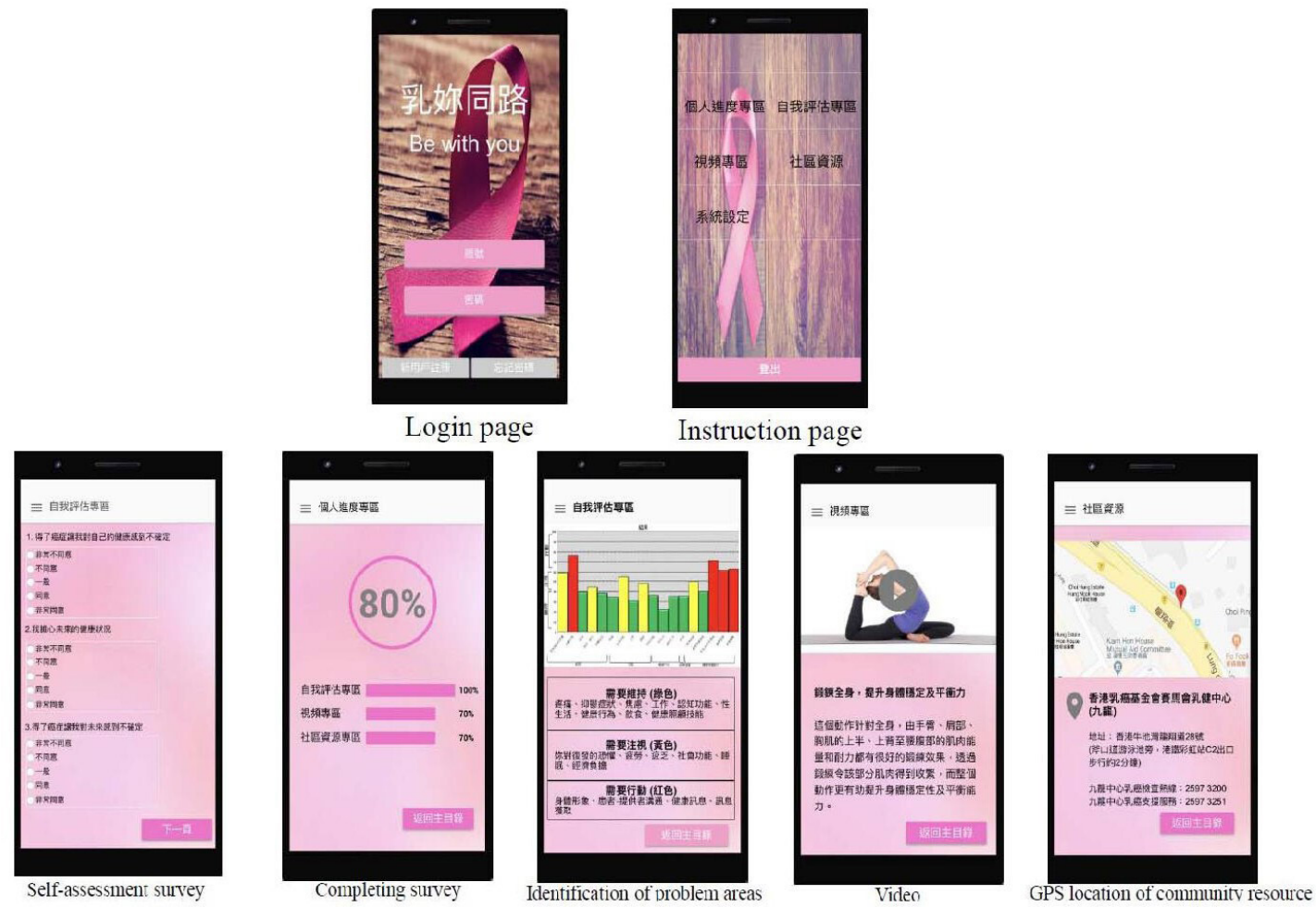

Figure 1 Functionality of Be-with-You app.

there are about 2500 new cases of breast cancer in this hospital.

\section{Inclusion criteria}

The inclusion criteria require that participants: (1) are female; (2) are breast cancer survivors after treatment and discharged from the Hunan Cancer Hospital; (3) have cancer that is stage I, II or III; (4) have completed primary treatment within 1 year or longer but less than 3 years; (5) are between 18 and 65 years of age; (6) have an acceptable literacy level (eg, are able to read and write Chinese); (7) have a smartphone; (8) are agreeable to the study requirement of monitoring their mobile app's usage log; and (9) are able to provide informed consent.

\section{Exclusion criteria}

The exclusion criteria require that participants: (1) have reported history of severe mental illness including schizophrenia, bipolar depression, and so on; (2) have no cancer diagnosis other than breast cancer; (3) have no previous participation in clinical trial; and (4) have not used another self-management app to manage breast cancer short or long-term effects.

\section{Outcomes}

Primary and secondary outcomes will be measured for all participants at the study's outset (for baseline data), and again at 4 weeks and 12 weeks after intervention. All measurements are selected based on their satisfactory construct validity and reliability. A flow chart of screening, enrolment, interventions and assessments is shown in figure 2.

\section{Primary outcomes}

Health literacy

The Brief Health Literacy Screening is a three-item selfrated questionnaire that aims to detect the health literacy level of patients. All items are ranked on a 5-point rating scale, with total scores ranging from 3 to 15 . A higher score indicates higher health literacy. ${ }^{36}$

\section{Problem-solving skills}

The Social Problem-Solving Inventory Revised-Short Form (SPSI-R:S) is a 25-item self-assessment measure that evaluates an individual's problem-solving orientation and problem-solving performance. It is derived from the Social Problem-Solving Inventory Revised-Full Form, which consisted of 52 items. ${ }^{37}$ The SPSI-R:S consists of five subscales: positive problem orientation (PPO), negative problem orientation (NPO), rational problem solving (RPS), impulsivity/carelessness style (ICS) and avoidance style (AS). All items are rated on a 5-point Likert scale, with total scores ranging from 0 to 100 . High scores on PPO and RPS and low scores on NPO, ICS and AS indicate good social problem-solving skills, and low PPO and RPS scores and high NPO, ICS and AS scores indicate poor social problem-solving skills. ${ }^{38}$

\section{Self-management skills}

One self-management skill will be assessed by the Brief Medication Adherence Scale, which is a 10-item selfassessment measure of medication adherence behaviour. It was designed to be administered to examine an individual's attitudes towards taking medication and medication 
Eligibility screening

Enrolment

Week 0
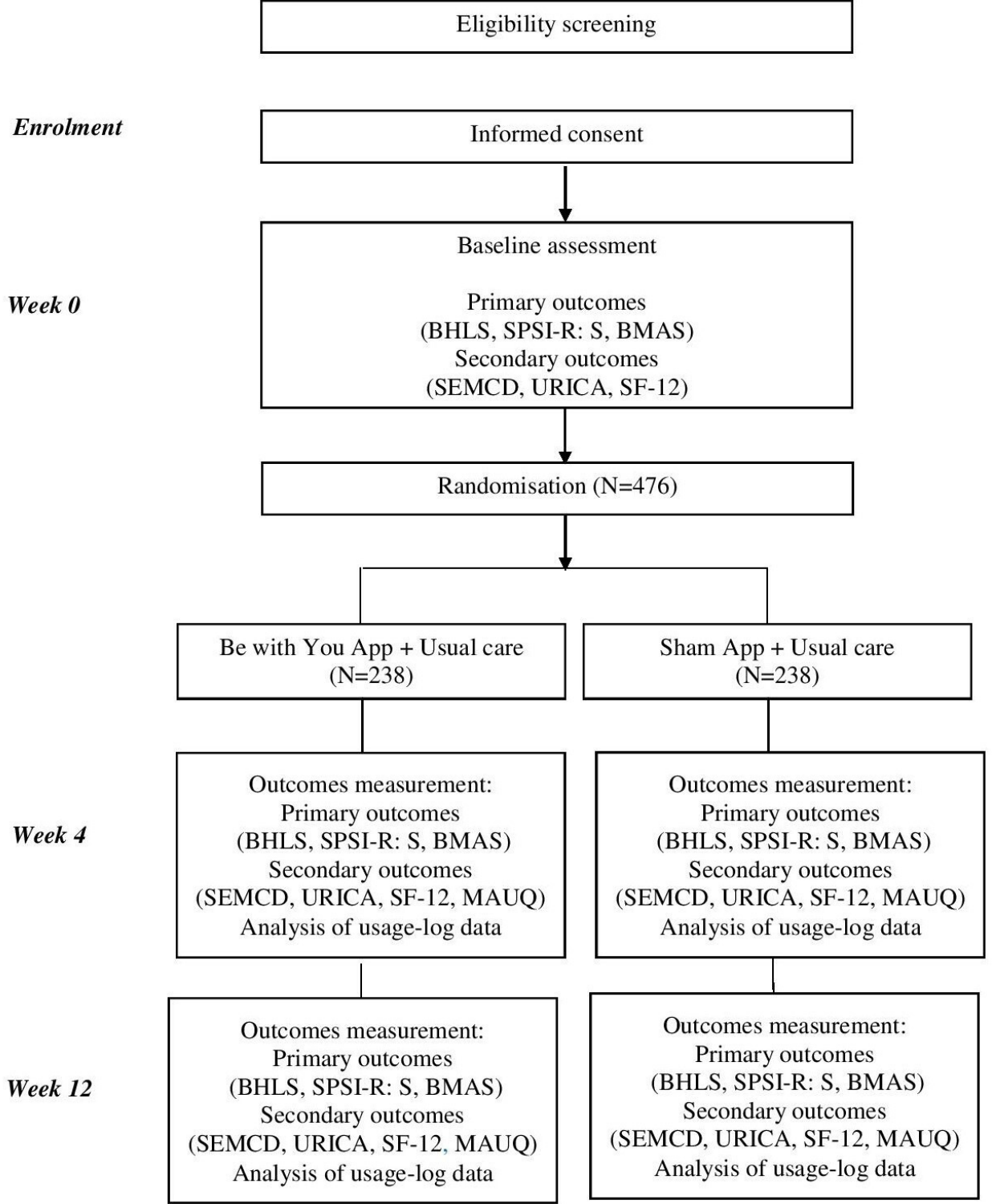

Figure 2 RCT flow diagram. BHLS, Brief Health Literacy Screening; BMAS, Brief Medication Adherence Scale; MAUQ, mHealth App Usability Questionnaire; SEMCD, Self-Efficacy to Manage Chronic Disease Scale; SF-12, 12-item Short-Form Health Survey; SPSI-R:S, Social Problem-Solving Inventory Revised-Short Form; URICA, University of Rhode Island Change Assessment Scale.

adherence behaviour during the past 2 weeks. ${ }^{39}$ Each item is rated on a 10 -point scale ranging from 1 to 10 , resulting in a score range of $10-100$ points for answering all 10 items. A higher score indicates higher level of medication adherence. ${ }^{39}$

\section{Secondary outcomes}

Self-efficacy

The Self-Efficacy to Manage Chronic Disease Scale is a six-item scale that has been developed for assessing an individual's self-efficacy for managing chronic disease and evaluates the effectiveness of chronic disease selfmanagement programmes. ${ }^{40}$ Each item is rated on a 10-point scale, and the total score is the mean of the scale's six items and ranges from 1 to 10 . A higher score indicates higher self-efficacy. The test has been demonstrated to have good validity and reliability in multiple studies. $^{41}$

\section{Readiness for change}

The University of Rhode Island Change Assessment Scale is a 32-item self-report measure of readiness for change that is based on the transtheoretical therapy model. ${ }^{42}$ The scale consists of four subscales that represent four stages of change (precontemplation, contemplation, action and maintenance). All items are rated on a 5-point Likert scale, with 1 representing strongly disagree and 5 strongly agree. The total score of each subscale ranges from 7 to 35. Profile scores and readiness to change can be generated. $^{43}$ 


\section{Health-related quality of life}

The 12-item Short-Form Health Survey (SF-12) is a simplified version of the Medical Outcomes Study 36-Item ShortForm Health Survey. ${ }^{44}$ The Chinese version of the SF-12 is a validated generic HRQoL instrument containing 12 items. It measures HRQoL through a physical component summary domain and a mental component summary domain, with higher scores reflecting better HRQoL. ${ }^{45}$

\section{Adherence to the mobile app}

With reference to previous studies, usage log data will be collected using the apps' usage monitoring system and will be assessed at 4 weeks and 12 weeks after intervention. The user logs will contain time stamps for each function that are recorded whenever a participant uses that function. ${ }^{46} 47$

\section{Perceived usability of the mobile app}

The mHealth App Usability Questionnaire for standalone mHealth app (patient version) is an 18-item scale to measure user's perceived usability and satisfaction of the mobile app. It measures three domains, namely ease of use (five items), interface and satisfaction (seven items) and usefulness (six items). All items are rated on a 7-point Likert scale, with 1 representing strongly agree and 7 strongly disagree. A lower score indicates higher perceived usability. ${ }^{4}$

\section{Statistical plan}

\section{Power and sample size calculations}

The sample size was calculated using the statistical power analysis program $\mathrm{G}^{*}$ power V.3.1.0, with the alpha level at 0.05 and a study power of $80 \%$. Participants in intervention groups in previous studies achieved a significant difference in their usability of an app, their quality of life and their social problem-solving skills when compared with the control subjects (effect sizes ranged from 0.2765 to 1.8533$).{ }^{49}{ }^{50}$ Thus, a smallest effect size of 0.2765 is estimated for between-group comparisons of the primary outcomes in this study, so the sample size will be 414 . Presuming a dropout rate of 15\%, 62 additional participants will be recruited. Thus, the planned sample size will be 476 , with 238 participants per group. In addition, we will have three research students to help follow-up the participants in order to minimise the dropout rate. That is a feasible sample size for a clinical trial with the required budget and proposed time frame of this study.

\section{Data analysis}

All statistical analyses will be completed using the IBM SPSS Version 24statistical software package with a 5\% level of significance (two sided). The data will be double entered for validation purposes. Data on all outcome measures will be analysed on an intention-to-treat basis. The baseline characteristics of participants between the two groups will be compared using two samples and independent t-tests, $\chi^{2}$ tests or Fisher's exact test, as appropriate. Mixed effects models will be used to compare the differential changes between the two groups in all outcome measures at different points in time. The strength of a mixed effects model is that it accounts for intracorrelation between repeatedly measured data and accommodates missing data caused by dropouts, provided that the missing data are random. Two different approaches will be used to analyse usage log data: the random effects model will be used to observe changes in the app usage levels over time, and the Cox proportional hazards model will be used to observe changes in the probability of stopping use of the app.

\section{ETHICS AND DISSEMINATION}

The study's design, research ethics and safety considerations have been thoroughly examined. Ethical approval was granted by The Hong Kong Polytechnic University. The trial has been registered on Chinese Clinical Trail Registry (http://www.chictr.org.cn/searchprojen.aspx). It is estimated that the trial will be completed in 2021, and the data analysis and dissemination of the results will be published afterwards.

\section{Author affiliations}

${ }^{1}$ Department of Rehabilitation Sciences, The Hong Kong Polytechnic University, Kowloon, Hong Kong

${ }^{2}$ Department of Nursing, Hunan Cancer Hospital, Changsha, China

${ }^{3}$ Department of Computing, The Hong Kong Polytechnic University, Kowloon, Hong Kong

${ }^{4}$ The Third Affiliated Hospital of Guangzhou Medical University, Guangzhou, China

${ }^{5}$ Consultant in Cancer Survivorship, Gaithersburg, Maryland, USA

${ }^{6}$ Hunan Cancer Hospital, The Affiliated Cancer Hospital of Xiangya School of Medicine, Central South University, Changsha, China

Acknowledgements The authors thank Professor Yongyi Chen, PhD, Department of Hospital Office, Hunan Cancer Hospital/The Affiliated Cancer Hospital of Xiangya School of Medicine, Central South University, for her support to conduct the study at Hunan Cancer Hospital.

Contributors ASKC, XL and MF conceived the study, developed the study design and drafted the manuscript. PHFN developed the study design, and provided expertise in the development of two apps. CTTK and YZ participated in the study design, and in particular in the definition of outcomes. All authors contributed to the refinement of the study protocol and approved the final manuscript.

Funding This project has been funded by the Department of Rehabilitation Sciences, The Hong Kong Polytechnic University (project number ZVMW).

Competing interests None declared.

Patient and public involvement Patients and/or the public were involved in the design, or conduct, or reporting, or dissemination plans of this research. Refer to the Methods section for further details.

Patient consent for publication Not required.

Provenance and peer review Not commissioned; externally peer reviewed.

Open access This is an open access article distributed in accordance with the Creative Commons Attribution Non Commercial (CC BY-NC 4.0) license, which permits others to distribute, remix, adapt, build upon this work noncommercially, and license their derivative works on different terms, provided the original work is properly cited, appropriate credit is given, any changes made indicated, and the use is non-commercial. See: http://creativecommons.org/ licenses/by-nc/4.0/.

ORCID iDs

Andy S K Cheng http://orcid.org/0000-0001-7503-5273

Yingchun Zeng http://orcid.org/0000-0002-1525-6725 


\section{REFERENCES}

1 Ferlay J, Ervik M, Lam F, et al. Global cancer observatory: cancer today. Lyon, France: International Agency for Research on Cancer, 2018.

2 Zeng $\mathrm{H}$, Chen $\mathrm{W}$, Zheng $\mathrm{R}$, et al. Changing cancer survival in China during 2003-15: a pooled analysis of 17 population-based cancer registries. Lancet Glob Health 2018;6:e555-67.

3 Tan X, Liu X, Shao H, et al. Healthy China 2030: a vision for health care. Value Health Reg Issues 2017;12:112-4.

4 Burg MA, Adorno G, Lopez EDS, et al. Current unmet needs of cancer survivors: analysis of open-ended responses to the American cancer Society study of cancer survivors II. Cancer 2015;121:623-30.

5 So WKW, Chow KM, Chan HYL, et al. Quality of life and most prevalent unmet needs of Chinese breast cancer survivors at one year after cancer treatment. Eur J Oncol Nurs 2014;18:323-8.

6 Wang J-W, Shen Q, Ding N, et al. A qualitative exploration of the unmet psychosocial rehabilitation needs of cancer survivors in China. Psychooncology 2016;25:905-12.

7 Gapstur RL. Symptom burden: a concept analysis and implications for oncology nurses. Oncol Nurs Forum 2007;34:673-80.

8 Taghian NR, Miller CL, Jammallo LS, et al. Lymphedema following breast cancer treatment and impact on quality of life: a review. Crit Rev Oncol Hematol 2014;92:227-34.

9 Harrington CB, Hansen JA, Moskowitz M, et al. It's not over when it's over: long-term symptoms in cancer survivors--a systematic review. Int J Psychiatry Med 2010;40:163-81.

10 Montazeri A, Vahdaninia M, Harirchi I, et al. Quality of life in patients with breast cancer before and after diagnosis: an eighteen months follow-up study. BMC Cancer 2008;8:330.

11 Reyes-Gibby CC, Anderson KO, Morrow PK, et al. Depressive symptoms and health-related quality of life in breast cancer survivors. J Womens Health 2012;21:311-8.

12 Deshields TL, Potter P, Olsen S, et al. The persistence of symptom burden: symptom experience and quality of life of cancer patients across one year. Support Care Cancer 2014;22:1089-96.

13 Chow R, Pulenzas N, Zhang L, et al. Quality of life and symptom burden in patients with breast cancer treated with mastectomy and lumpectomy. Support Care Cancer 2016;24:2191-9.

14 Zeng YC, Cheng ASK, Liu XY, et al. Symptom profiles, work productivity and quality of life among Chinese female cancer survivors. Obstetrics \& Gynecology 2016:1-6.

15 Kim HS, Park JS, Kim HS, et al. Self-Management needs of breast cancer survivors after treatment: results from a focus group interview. Cancer Nurs 2018.

16 Grover A, Joshi A. An overview of chronic disease models: a systematic literature review. Glob J Health Sci 2014;7:210-27.

17 Kadu MK, Stolee P. Facilitators and barriers of implementing the chronic care model in primary care: a systematic review. BMC Fam Pract 2015;16:12.

18 Lorig KR, Holman H. Self-Management education: history, definition, outcomes, and mechanisms. Ann Behav Med 2003;26:1-7.

19 Kulinski KP, Boutaugh M, Smith ML, et al. Setting the stage: measure selection, coordination, and data collection for a national selfmanagement initiative. Front Public Health 2014;2:206.

20 Haslbeck J, Zanoni S, Hartung U, et al. Introducing the chronic disease self-management program in Switzerland and other Germanspeaking countries: findings of a Cross-border adaptation using a multiple-methods approach. BMC Health Serv Res 2015;15:576.

21 Risendal B, Dwyer A, Seidel R, et al. Adaptation of the chronic disease self-management program for cancer survivors: feasibility, acceptability, and lessons for implementation. J Cancer Educ 2014;29:762-71.

22 Davy C, Bleasel J, Liu H, et al. Effectiveness of chronic care models: opportunities for improving healthcare practice and health outcomes: a systematic review. BMC Health Serv Res 2015;15:194.

23 Whitehead L, Seaton P. The effectiveness of self-management mobile phone and tablet apps in long-term condition management: a systematic review. J Med Internet Res 2016;18:E97.

24 Schweitzer J, Synowiec C. The economics of eHealth and mHealth. J Health Commun 2012;17 Suppl 1:73-81.

25 Quintiliani LM, Mann DM, Puputti M, et al. Pilot and feasibility test of a mobile health-supported behavioral counseling intervention for weight management among breast cancer survivors. JMIR Cancer 2016;2:e4.

26 Fu MR, Axelrod D, Guth AA, et al. Usability and feasibility of health it interventions to enhance self-care for lymphedema symptom management in breast cancer survivors. Internet Interv 2016;5:56-64.
27 Baseman J, Revere D, Baldwin L-M. A mobile breast cancer survivorship care APP: pilot study. JMIR Cancer 2017;3:e14.

28 Kuijpers W, Groen WG, Oldenburg HS, et al. eHealth for breast cancer survivors: use, feasibility and impact of an interactive portal. JMIR Cancer 2016;2:e3-11.

29 Lozano-Lozano M, Galiano-Castillo N, Martín-Martín L, et al. Monitoring energy balance in breast cancer survivors using a mobile APP: reliability study. JMIR Mhealth Uhealth 2018;6:e67.

30 Lozano-Lozano M, Cantarero-Villanueva I, Martin-Martin L, et al. A mobile system to improve quality of life via energy balance in breast cancer survivors (BENECA mHealth): prospective testretest Quasiexperimental feasibility study. JMIR Mhealth Uhealth 2019;7:e14136

31 Seiler A, Klaas V, Tröster G, et al. eHealth and mHealth interventions in the treatment of fatigued cancer survivors: a systematic review and meta-analysis. Psychooncology 2017;26:1239-53.

32 Haberlin C, O'Dwyer T, Mockler D, et al. The use of eHealth to promote physical activity in cancer survivors: a systematic review. Support Care Cancer 2018;26:3323-36.

33 Cheng ASK, Liu X, Kwok CTT, et al. Chinese translation of a measure of symptom burden, functional limitations, lifestyle, and health careseeking skills in breast cancer survivors: the cancer Survivor profile. J Cancer Surviv 2019;13:130-47.

34 Todd BL, Feuerstein M, Gehrke A, et al. Identifying the unmet needs of breast cancer patients post-primary treatment: the cancer Survivor profile (CSPro). J Cancer Surviv 2015;9:137-60.

35 Gehrke A, Lee SS, Hilton K, et al. Development of the cancer Survivor Profile-Breast cancer (CSPro-BC) APP: patient and nurse perspectives on a new navigation tool. $J$ Cancer Surviv 2018;12:291-305.

36 Wang C, Lang J, Xuan L, et al. The effect of health literacy and self-management efficacy on the health-related quality of life of hypertensive patients in a Western rural area of China: a crosssectional study. Int J Equity Health 2017;16:58.

37 D'Zurilla TJ, Nezu AM, Maydeu-Olivares A, et al. SPSI-R): technical manual. North Tonawanda, NY: Multi-Health Systems, Inc, 2002.

38 Siu AMH, Shek DTL. The Chinese version of the social problemsolving inventory: some initial results on reliability and validity. J Clin Psychol 2005;61:347-60.

39 Chui E, Wong KL, Chan KY, et al. Validation study of the brief medication adherence scale (BMAS) in patients with schizophrenia and related disorders in Hong Kong. Asian J Psychiatr 2018;37:154-60.

$40 \mathrm{Hu} \mathrm{H}$, Li G, Arao T. Validation of a Chinese version of the selfefficacy for managing chronic disease 6-Item scale in patients with hypertension in primary care. ISRN Public Health 2013;2013:1-6.

41 Ritter PL, Lorig K. The English and Spanish self-efficacy to manage chronic disease scale measures were validated using multiple studies. J Clin Epidemiol 2014;67:1265-73.

42 McConnaughy EA, DiClemente CC, Prochaska JO, et al. Stages of change in psychotherapy: a follow-up report. Psychotherapy: Theory, Research, Practice, Training 1989;26:494-503.

43 Chan SCC, Chan $\mathrm{CCH}$, Siu AMH, et al. Stage of change in selfmanagement of chronic diseases: psychometric properties of the Chinese version of the University of Rhode island change assessment (C-URICA) scale. Rehabil Psychol 2007;52:103-12.

44 Ware J, Kosinski M, Keller SD. A 12-Item short-form health survey: construction of scales and preliminary tests of reliability and validity. Med Care 1996;34:220-33.

45 Lam CLK, Tse EYY, Gandek B. Is the standard SF-12 health survey valid and equivalent for a Chinese population? Qual Life Res 2005;14:539-47

46 Lee $\mathrm{K}$, Kwon $\mathrm{H}$, Lee $\mathrm{B}$, et al. Effect of self-monitoring on long-term patient engagement with mobile health applications. PLoS One 2018;13:e0201166.

47 Bradway M, Pfuhl G, Joakimsen R, et al. Analysing mHealth usage logs in RCTs: explaining participants' interactions with type 2 diabetes self-management tools. PLoS One 2018;13:e0203202.

48 Zhou L, Bao J, Setiawan IMA, et al. The mHealth APP usability questionnaire (MAUQ): development and validation study. JMIR Mhealth Uhealth 2019;7:e11500.

$49 \mathrm{Ma} \mathrm{J}$, Xiao L, Blonstein AC. Measurement of self-monitoring web technology acceptance and use in an e-health weight-loss trial. Telemed J E Health 2013;19:739-45.

50 Sahler OJZ, Varni JW, Fairclough DL, et al. Problem-Solving skills training for mothers of children with newly diagnosed cancer: a randomized trial. J Dev Behav Pediatr 2002;23:77-86. 